\title{
Genome-wide expression profiling; a panel of mouse tissues discloses novel biological functions of liver $\mathbf{X}$ receptors in adrenals
}

\author{
Knut R Steffensen, Soek Ying Neo', Thomas M Stulnig ${ }^{2}$, Vinsensius B Vega ${ }^{1}$, \\ Safia S Rahman ${ }^{1}$, Gertrud U Schuster, Jan-Åke Gustafsson and Edison T Liu ${ }^{1}$ \\ Department of Biosciences, Karolinska Institutet at NOVUM, Huddinge, Sweden \\ ${ }^{1}$ Genome Institute of Singapore, 60 Biopolis Street, The Genome, \#02-01, Singapore 138672 \\ 2Division of Endocrinology and Metabolism, Department of Internal Medicine III, University of Vienna, and CeMM - Center of Molecular Medicine, \\ Austrian Academy of Sciences, Vienna, Austria \\ (Requests for offprints should be addressed to K R Steffensen; Email: knut.steffensen@ biosci.ki.se)
}

\begin{abstract}
The liver $X$ receptors $\alpha$ and $\beta$ (LXR $\alpha$ and LXR $\beta$ ) are members of the nuclear receptor superfamily of proteins which are highly expressed in metabolically active tissues. They regulate gene expression of critical genes involved in cholesterol catabolism and transport, lipid and triglyceride biosynthesis and carbohydrate metabolism in response to distinct oxysterols and intermediates in the cholesterol metabolic pathway. The biological roles of the LXRs in tissues other than liver, intestine and adipose tissue are poorly elucidated. In this study we used global gene-expression profiling analysis to detect differences in expression patterns in several tissues from mice fed an LXR agonist or vehicle. Our results show that LXR plays an important role in the kidney, lung, adrenals, brain, testis and heart where several putative LXR target genes were found. The effects of the LXRs were further analysed in adrenals where treatment with an LXR agonist induced expression of adrenocorticotrophic hormone receptor, suppressed expression of uncoupling protein (UCP)-1 and UCP-3 as well as several glycolytic enzymes and led to increased serum corticosterone levels. These results indicate novel biological roles of the LXR including regulation of energy metabolism, glycolysis and steroidogenesis in the adrenals via alteration of expression profiles of putative target genes.
\end{abstract}

Journal of Molecular Endocrinology (2004) 33, 609-622

\section{Introduction}

The liver $\mathrm{X}$ receptor (LXR) subfamily of nuclear receptors consists of two members, $\operatorname{LXR} \alpha$ and LXR $\beta$, which are activated upon binding of ligand. Endogenous ligands most probably comprise distinct oxysterols, namely 20(S)- and 22(R)hydroxycholesterol and 20,22-dihydroxycholesterol (Janowski et al. 1996). Together with the retinoid X receptor (RXR), LXR binds as an LXR-RXR heterodimer to specific DR4 response elements (direct hexameric repeats spaced by four nucleotides) in promoters of their cognate target genes (Willy et al. 1995). Whereas LXR $\beta$ is expressed ubiquitously, high expression of $\mathrm{LXR} \alpha$ is restricted to liver, adipose tissue, small intestine and macrophages (Willy et al. 1995, Auboeuf et al. 1997). The LXRs have recently gained much attention for their role in cholesterol, lipid and carbohydrate metabolism (reviewed in Steffensen and Gustafsson 2004).

The LXRs play a pivotal role in cholesterol and lipid metabolism by various mechanisms. LXR $\alpha^{-/-}$but not $\operatorname{LXR} \beta^{-/-}$mice show significant increase in hepatic cholesterol content (Alberti et al. 2001), and pronounced changes in blood lipid profiles have been observed in

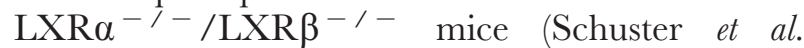
2002). LXRs promote lipogenesis by inducing transcription of several key lipogenic enzymes 
and $\operatorname{LXR} \alpha^{-/-} / \mathrm{LXR}^{-/-}$mice have less adipose tissue than their wild-type littermates (Juvet et al. 2003). The ATP-binding cassette (ABC) transporters ABCA1 and ABCG1 facilitate cholesterol efflux from, for example, macrophages thereby preventing foam cell formation and atherogenesis. Apolipoprotein E (apoE) is another crucial player in reverse cholesterol transport, facilitating incorporation of cholesterol into highdensity lipoprotein particles for transport to the liver. Both the ABCA1/G1/G5/G8 transporters (Costet et al. 2000, Kennedy et al. 2001, Repa et al. 2002) and the apoE/C-I/C-IV/C-II gene cluster (Mak et al. 2002) are induced by the LXRs, indicating important roles of LXR in suppression of atherosclerosis. Expression of gluconeogenic enzymes was significantly downregulated in livers of animals treated with an LXR agonist (Stulnig et al. 2002a, 2002b). Suppression of gluconeogenetic enzymes in liver, induction of GLUT4 and improved glucose tolerance by activation of LXR have also been reported (Dalen et al. 2003, Laffitte et al. 2003). Another study showed reduced blood glucose levels in diabetic rodents, increased insulin sensitivity in insulin-resistant Zucker rats and expression of gluconeogenic genes were suppressed leading to decreased hepatic glucose output upon treatment of an LXR agonist (Cao et al. 2003).

The findings described above suggest that the main target tissues of the LXRs are liver and adipose tissue where their function has been carefully studied and the results strongly indicate important roles of LXRs in nutrient metabolism. As most of the LXR target genes have been identified in liver, intestines and adipose tissue, the role of the LXRs in other tissues remains obscure. In this study, we have analysed the role of the LXRs in several tissues by genome-wide expression-profiling analysis of seven organs including liver, lung, kidney, brain, testis, adrenal and heart from mice treated orally with a synthetic LXR agonist. Changes in expression profiles due to treatment observed in wild-type mice were compared with those observed in $\mathrm{LXR} \alpha^{-/-} / \mathrm{LXR} \beta^{-/-}$mice to ensure LXRspecific regulation. Beyond confirming earlier results and identifying additional candidate target genes of LXR in lipid and cholesterol metabolism, we disclose unknown putative LXR target genes suggesting novel biological functions of the LXRs in lung, kidney, brain, testis, adrenal and heart.

\section{Materials and methods}

\section{Animal treatment}

LXR $\alpha^{-/-} / \mathrm{LXR}^{-/-}$double-targeted knockout mice were generated in our laboratory as described previously (Schuster et al. 2002). The mice used in this study were male Sv129/C57BL/6 hybrids backcrossed in C57BL/6 mice for three generations. Mice were housed under a 12-h/12-h light/dark cycle in the specific pathogen-free animal unit at the Karolinska University Hospital in Huddinge, Sweden. For the experiment, mice were 10-12 months of age, had free access to water and an experimental diet based on a low-fat standard rodent diet (R36; Lactamin AB, Vadstena, Sweden). The diet was either mixed with vehicle alone (ethanol) or supplemented with $0.025 \% \quad(\mathrm{w} / \mathrm{w})$ of the synthetic LXR agonist T0901317 (referred to hereon as T1317; Repa et al. 2000) and was dried extensively to evaporate any traces of ethanol. Four wild-type and four $\mathrm{LXR} \alpha^{-/-} / \mathrm{LXR} \beta^{-/-}$mice were assigned to each experimental group, except for liver where three animals were used, and treated for 7 days. Mice were killed and tissues snap-frozen in liquid nitrogen and kept at $-80{ }^{\circ} \mathrm{C}$ until isolation of RNA. The experiments were approved by the local ethical committee for animal experiments and the guidelines for the use and care of laboratory animals followed.

\section{Microarray experiments}

RNA was linearly amplified for two rounds using a procedure modified from Eberwine et al. (1992). Total RNA was reverse-transcribed using a 63-nucleotide synthetic primer containing the T7 polymerase-binding site: 5'-GGCCAGTGAA TTGTAATACGACTCACTATAGGGAGGGGG $(\mathrm{T})_{24}-3^{\prime}$. Full-length double-stranded cDNA synthesis was performed in the presence of Escherichia coli DNA polymerase I, DNA ligase and RNase H. The cDNA was made blunt-ended with T4 DNA polymerase, and purified by extraction in a mixture of phenol, chloroform and isoamyl alcohol, and precipitation in the presence of ammonium acetate and ethanol. Purified double-stranded 
cDNA was then transcribed with T7 polymerase (T7 Megascript kit; Ambion, Austin, TX, USA) to yield linearly amplified antisense RNA, which was subsequently purified with RNeasy mini-columns (Qiagen).

For the second round of amplification, an aliquot of the amplified RNA was reverse-transcribed to cDNA in the presence of random hexamer oligonucleotides. Second-strand cDNA synthesis was initiated in the presence of oligo(dT) T7 primer containing the T7 polymerase-binding site (as described above) in the presence of $E$. coli DNA polymerase I, DNA ligase and RNase H. The purification of cDNA and in vitro transcription was carried out as before. Mouse universal reference RNA (Stratagene), which comprised of total RNA from 11 different mouse cell lines, was amplified and used as the reference for cDNA microarray analysis.

A total of approximately 10000 mouse cDNA features (Incyte Genomics, Palo Alto, CA, USA) were spotted on to poly-L-lysine-coated slides using the OmniGrid arrayer (GeneMachines, Ann Arbor, MI, USA). Probes were generated from the amplified RNA material and hybridized to the chip as described elsewhere (Sotiriou et al. 2002). Briefly, $4 \mu \mathrm{g}$ amplified RNA was reverse-transcribed using random hexamers and labelled directly with Cy3-conjugated dUTP (reference RNA) or Cy5conjugated dUTP (sample RNA). Hybridization was performed in the presence of $25 \%$ formamide and $5 \times \mathrm{SSC}$ for $16 \mathrm{~h}$ at $42{ }^{\circ} \mathrm{C}$. Slides were scanned with an Axon 4000B laser scanner (Axon Instruments, Union City, CA, USA) after washing and drying. To minimize the effects of labelling biases, reciprocal dye-swap labelling experiments were performed for each individual mouse tissue sample. Genes found to be regulated in $\mathrm{LXR} \alpha^{-/-} / \mathrm{LXR}^{-/-}$mice upon LXR agonist feeding as well as in wild-type mice were subtracted and are not presented as putative LXR target genes.

\section{Data analysis}

Raw data were analysed on GenePix analysis software version $4 \cdot 0$ (Axon Instruments) and uploaded to a relational database. The cDNA clones used for the microarray are represented by their UniGene identifiers. The logarithmic expression ratio for a spot on each array was normalized by subtracting the median logarithmic ratio for the same array. Data were filtered to exclude spots with a size of less than $25 \mu \mathrm{m}$ and any poor-quality or missing spots. Gene features that were found to be absent from the data in more than $50 \%$ of the arrays for each tissue were also excluded. Application of these filters resulted in the inclusion of between 9400 and 9600 genes in the subsequent analyses. This approach was based on the rationale of including as many data points as possible to capture maximal information in the early stage of the analysis. The correlation of the overall data from reciprocal labelling was good (approximately 0.8 on average). To identify outlier genes due to treatment in each group of animals (wild-type and knockout mice), the replicates of each individual mouse tissue sample (reciprocal dye-swap-labelled experiments) were treated as individual data points and the Wilcoxon rank-sum non-parametric test was applied. Genes were identified as putative LXR target genes if they were only found to be outliers due to treatment specifically in wild-type mice $(P<0 \cdot 01)$. To evaluate gene-expression patterns by hierarchical clustering, the pair of replicates for each mouse tissue specimen was averaged and treated as a single data point, and clustering using Pearson's uncentred correlation metric and average linkage (Eisen et al. 1998) was performed on normalized data $($ mean $=0$, S.D. $=1)$ of the averaged values. Genes that showed at least $1 \cdot 3$-fold change in expression $(P<0 \cdot 01)$ were selected for further downstream analyses (see Results). The data set and annotated list of putative LXR target genes are available for downloading as additional supplementary material at http://www.gis.a-star.edu.sg/supplmat/neosy/ LXRmouse.html.

\section{Quantitative-PCR (Q-PCR) and primer designs}

Total RNA was isolated from tissues using Trizol reagent (Invitrogen) according to the manufacturer's instructions. RNA was further purified by the RNeasy kit (Qiagen) and checked for quality by spectrophotometry at $260 / 280 \mathrm{~nm}$ and $1.5 \%$ agarose gel electrophoresis. A total of $1 \mu \mathrm{g}$ RNA was treated with DNase I (amplification grade; Invitrogen) before reverse transcription into cDNA by Superscript II (Invitrogen) using random hexamer priming according to the manufacturer's instructions. mRNA expression was quantified with SYBRgreen chemistry on an ABI 7700 machine 
(Applied Biosystems) and a direct comparative method was used for data analysis. Expression of the gene of interest were normalized to the $18 \mathrm{~S}$ internal standard. All Q-PCR reactions were run with $20 \mathrm{ng}$ cDNA as template except for the $18 \mathrm{~S}$ internal control where $40 \mathrm{pg}$ cDNA were used. Primer sequences for the analysed genes were designed using PrimerExpress 1.5a (Applied Biosystems) and all sequences are available upon request. The efficiency of the Q-PGR reaction for $18 \mathrm{~S}$ and the genes of interest was $95-100 \%$, calculated using a standard curve and the following formula: $\mathrm{E}_{\text {fficiency of PCR }}=\left(10^{-1 / \text { slope of standard curve })}-1\right.$ (for more details see User Bulletin \#2, Applied Biosystems, www.appliedbiosystems.com). Student's $\mathrm{t}$ tests on average expression from the four mice $(n=4)$ were performed to determine the level of significance of the changes observed between wild-type vehicle and LXR T1317 agonist-treated animals and the $P$ value is indicated in the text or the respective Figure.

\section{Hormone analysis and statistics}

Serum corticosterone concentration in each mouse was determined by RIA as follows; $50 \mu \mathrm{l}$ serum were extracted with $1.0 \mathrm{ml}$ ethyl acetate and centrifuged at room temperature at $2000 \boldsymbol{g}$ for $5 \mathrm{~min} .100 \mu \mathrm{l}$ from the clear ethyl acetate phase was transferred in triplicates into polypropylene tubes, evaporated under vacuum and redissolved with assay buffer. RIA was carried out using tritiated corticosterone (New England Nuclear), anti-corticosterone (Sigma) and charcoal/dextran as a separating agent.

\section{Cell cultures}

Murine Y1 adrenocortical tumour and HIB-1B preadipocyte cells were maintained in Dulbecco's modified Eagle's medium (Art. no. 41965-039) $4500 \mathrm{~g} / \mathrm{l}$ glucose, supplemented with $10 \%$ fetal bovine serum, $2 \mathrm{mM}$ penicillin and $2 \mathrm{mM}$ streptomycin and maintained in a $5 \% \mathrm{CO}_{2}$ humidified atmosphere at $37^{\circ} \mathrm{C}$. HIB-1B cells were differentiated using the following protocol: cells were grown in standard growth medium and when cells reached tight confluence they were refed with the same medium supplemented with $18 \mathrm{nM}$ insulin, $1 \mathrm{nM}$ T3, $\quad 0.5 \mathrm{mM}$ isobutylmethyl-xanthine (IBMX), $0.5 \mu \mathrm{M}$ hydrocortisone and $0 \cdot 125 \mathrm{mM}$ indomethacin. Then, 3 days later, cells were placed back in standard growth medium in the presence of $18 \mathrm{nM}$ insulin, $1 \mathrm{nM}$ T3 and 0.5 $\mu \mathrm{M}$ BRL 49653 (a peroxisome proliferator-activated receptor (PPAR) $\gamma$ agonist) and incubated for an additional 4 days. Cell culture medium was replaced every $48 \mathrm{~h}$.

\section{Results}

To investigate functions of the LXR receptor isoforms in different tissues, we treated eight individual wild-type mice, four with the T1317 LXR agonist (Repa et al. 2000) and four with vehicle. Since the T1317 LXR agonist has been demonstrated to alter gene expression not only through the LXRs, but also via other unknown mechanisms (Stulnig et al. 2002b), LXR $\alpha^{-/-}$/ LXR $\beta^{-/-}$mice were also treated similarly to wild-type mice to identify gene responses solely mediated through LXR. At the end of the treatment, liver, kidneys, lungs, heart, brain, testis and adrenals were isolated from all mice and total RNA was extracted from the tissues and amplified for transcriptional profiling analyses on cDNA microarrays containing approximately 10000 gene features. Reciprocal dye-swap replicate hybridizations were performed to minimize technical noise. For each tissue, we investigated the differential patterns of gene expression between the vehicletreated group and the group fed with LXR agonist using the Wilcoxon rank-sum test, and asked whether genes that were differentially expressed upon treatment with the LXR agonist in the wild-type mice showed any change in expression in the $\operatorname{LXR} \alpha^{-/-} / \operatorname{LXR} \beta^{-/-}$mice.

Hierarchical clustering analyses based on the differentially expressed genes $(P<0 \cdot 01)$ showed distinct LXR agonist-induced changes in wild-type mice that were completely absent in $\operatorname{LXR} \alpha^{-/-}$/ LXR $\beta^{-/-}$mice in liver, adrenal, kidney, brain, testis, lung and heart tissues (see supplementary Fig. 1 on http://jme.endocrinology-journals.org/ content/vol33/issue3/). Induction of known target genes such as ABCA1/G1/G5, fatty acid synthase (FAS), lipoprotein lipase (LPL) and cholesterol $7 \alpha$-hydroxylase (Cyp7a1); (see Steffensen and Gustafsson 2004 and references therein) was detected upon LXR agonist treatment in the liver of wild-type mice. We also found downregulation of phosphoenolpyruvate carboxykinase (PEPCK) 


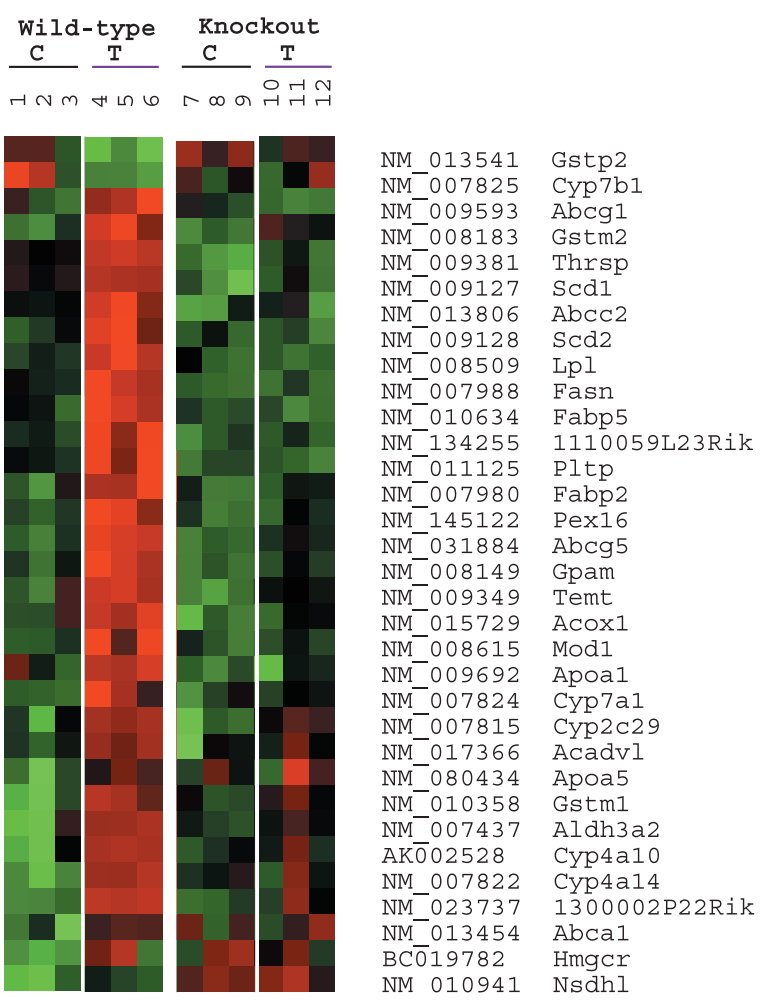

glutathione S-transferase, pi 2

cytochrome P450,7b1

ATP-binding cassette, sub-family G WHITE, member 1

glutathione $\mathrm{S}$-transferase, mu 2

thyroid hormone responsive SPOT14 homolog Rattus

stearoyl-Coenzyme A desaturase 1

ATP-binding cassette, sub-family C CFTR/MRP, member 2

stearoyl-Coenzyme $\mathrm{A}$ desaturase 2

lipoprotein lipase

fatty acid synthase

fatty acid binding protein 5, epidermal

Fatty acid elongase 1 homolog

phospholipid transfer protein

fatty acid binding protein 2, intestinal

peroxisome biogenesis factor 16

ATP-binding cassette, sub-family G WHITE, member 5

glycerol-3-phosphate acyltransferase, mitochondrial

thioether $\mathrm{S}$-methyltransferase

acyl-Coenzyme A oxidase 1, palmitoyl

malic enzyme, supernatant

apolipoprotein A-I

cytochrome P450, 7 al

cytochrome P450, 2c29

acyl-Coenzyme A dehydrogenase, very long chain apolipoprotein $\mathrm{A}-\mathrm{V}$

glutathione S-transferase, mu 1

aldehyde dehydrogenase family 3, subfamily A2

cytochrome P450, 4 a10

cytochrome P450, 4al4

Peroxisomal bifunctional enzyme

ATP-binding cassette, sub-family A ABC1, 1member

3-hydroxy-3-methylglutaryl-Coenzyme A reductase

NADP dependent steroid dehydrogenase-like

\section{suppression}

Figure 1 Gene-expression patterns in livers of wild-type and LXR $\alpha^{-/-/ L X R ~} \beta-/-$ mice upon T1317 LXR agonist treatment. Wild-type and LXR $\alpha^{-/-/ L X R \beta}{ }^{-/-}$mice were fed with either the T1317 LXR agonist or vehicle alone (three mice in each group) for 1 week and gene-expression profiling was performed on RNA extracted from the liver. An hierarchical cluster of selected genes that showed differential expression profiles between LXR agonist T1317 treated (lanes 4-6) and non-treated tissues (lanes 1-3) in wild-type mice $(P<0.01)$ is presented. For comparison, the same gene information from $L X R \alpha^{-/-/ L X R \beta}-/-$ mice (lanes 10-12 for the agonist-treated group and lanes 7-9 for the control group) is aligned. Rows represent individual genes, and columns represent unique samples. Each cell in the matrix represents the expression level of a gene feature in an individual tissue sample. The red and green bars reflect high and low expression levels respectively, while black indicates equivalent expression level. The magnitude of the log-transformed ratio is reflected by the degree of colour saturation.

expression in livers of LXR-agonist-treated mice as demonstrated previously (Stulnig et al. 2002b). However, the expression showed high variance and did not pass our selection criteria for inclusion in further annotations. These observations are in good concordance with the literature and also suggest that this approach is useful in discovering novel LXR target genes (Fig. 1). In wild-type mice, the largest number of LXR-regulated genes was found in liver and heart, followed by lung, adrenal, kidney, testis and brain (Fig. 2). Relatively more genes were induced upon treatment with an LXR agonist in liver and testis, while more genes were suppressed in kidney. The other tissues showed approximately equal numbers of up- and downregulated genes. The majority of tissues, except brain, exhibited fewer genes with altered expression profiles in the $\mathrm{LXR} \alpha^{-/-} / \mathrm{LXR} \beta^{-/-}$mice than those observed in the wild-type mice (Fig. 3) suggesting non-LXR-mediated effects of the T1317 LXR agonist since the transcriptional alterations in the $\mathrm{LXR} \alpha^{-/-} / \mathrm{LXR} \beta^{-/-}$mice cannot be due to LXR effects. A few genes showed altered expression in both wild-type and $\mathrm{LXR} \alpha^{-/-}$/ LXR $\beta^{-/-}$mice and these 'common' genes were excluded from further analyses of LXR-specific targets. Taken together, these data suggest the importance of LXR regulation in various tissues. 


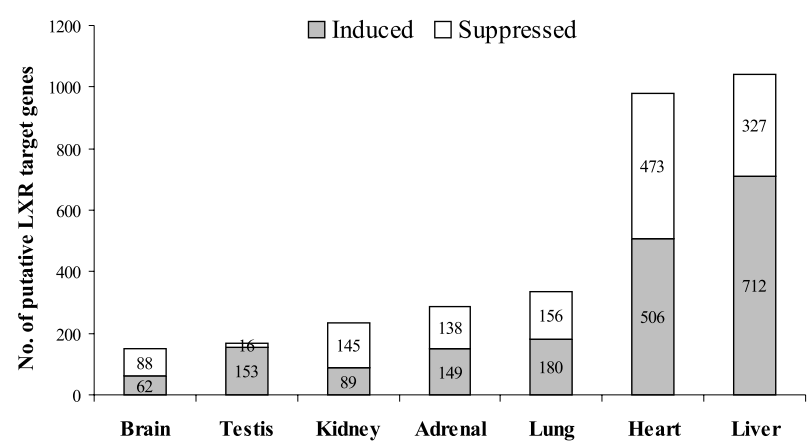

Figure 2 Summary of putative LXR target genes identified in each tissue. Genes showing significant $(P<0.01)$ induction (grey bars) and suppression (white bars) uniquely in wild-type mice upon LXR agonist treatment. The numbers of induced and suppressed genes are given within the bars for all seven tissues analysed. The selection criteria for the genes are described in detail in the Materials and methods section under Data analysis.

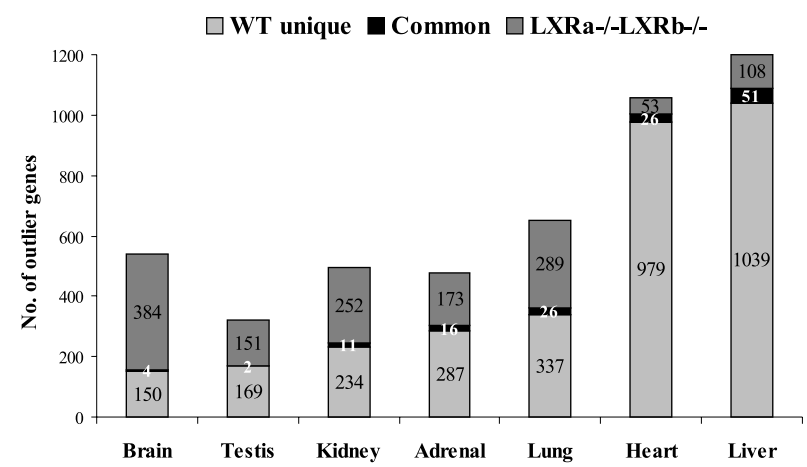

Figure 3 Summary of outlier genes found in each tissue in wild-type (WT) and knockout mice. Genes showing significantly $(P<0.01)$ altered expression uniquely in wild-type mice (light-grey bars), uniquely in $\mathrm{LXR} \alpha^{-/-} / \mathrm{LXR} \beta^{-/-}$mice (dark-grey bars) and in common in both groups (black bars). The number of genes in each category is given within the bars for each tissue analysed. The selection criteria for the genes are described in detail in the Materials and methods section under Data analysis.

Next we wanted to verify the observed changes in gene expression from the microarray experiment and performed Q-PCR on randomly selected genes in the liver, kidney, adrenal, lung and heart tissues. Fig. 4 shows that the expression of period homologue 2 (Per2; accession no. NM_011066), phosphatidic acid phosphatase type 2B (Pap2b; NM_080555), CDC-like kinase (Clk; NM_009905), apoE (NM_009696) and FAS (Fasn; AF127033) detected by Q-PCR were similar to that observed in the microarray, although the fold change was generally higher with the more sensitive Q-PGR technique. Furthermore, the expression of procollagen, type V, alpha 2 (Col5a2; NM_007737), which was observed to be either induced or suppressed in liver and adrenal, respectively, on microarray, was also verified by Q-PCR. These results indicate that the genes fulfilling our selection criteria are good candidates as putative LXR target genes.

To identify potential novel pathways involving LXRs, we selected putative LXR target genes $(P<0 \cdot 01)$ that showed at least $1 \cdot 3$-fold change in expression and characterized their biological functions based on SOURCE database at Stanford (http://source.stanford.edu/cgi-bin/sourceSearch). A comprehensive list of annotated genes with altered expression profiles upon LXR agonist treatment is available as additional supplementary material at http://www.gis.a-star.edu.sg/ supplmat/neosy/LXRmouse.html. A large number of genes with altered expression profiles were involved in lipid, cholesterol and carbohydrate metabolism, and these were found not only in liver (which was expected), but also in adrenal, kidney, lung and heart. Such induced genes included the known target gene ABCAl (Costet et al. 2000) and stearoyl CoA desaturases (SCD) 1 and 2, all of which are involved in fatty acid desaturation, and SPOT 14 and malic enzyme, involved in fatty acid metabolism. In addition to these known functions, LXR appears to be involved in a variety of previously unreported biological functions. The altered expression levels of several cytochrome P450 (Cyp450) enzymes, alcohol/aldehyde dehydrogenases and glutathione S-transferases indicate a possible involvement of LXR in detoxification processes and steroid metabolism. Changes in expression of several transcription factors, transcriptional cofactors and genes involved in splicing and translation were observed, particularly in liver and heart, suggesting that the LXRs may regulate transcription of some genes via indirect mechanisms in addition to direct regulation via their cognate response elements. Alterations in expression of several genes coding for serum proteins, coagulants/anticoagulants, proteases, protease inhibitors and signal-transduction molecules were also observed in the liver and adrenal. Taken together, these data indicate that the LXRs may have multiple biological functions in different tissues. 
Interestingly, several genes in the glycolysis pathway as well as protease inhibitors were suppressed in adrenals upon LXR agonist treat-

Liver

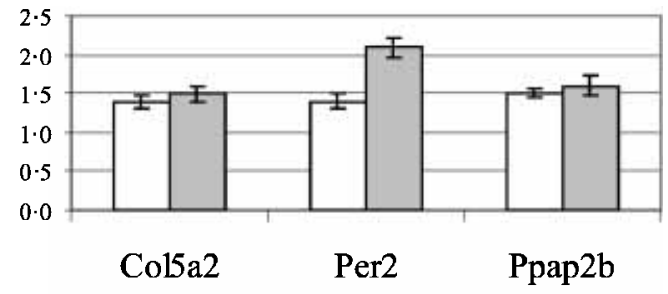

Kidney

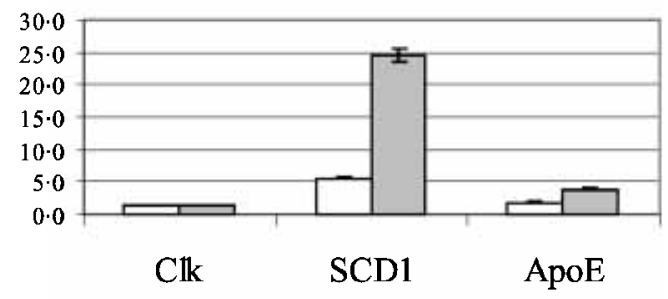

Adrenal

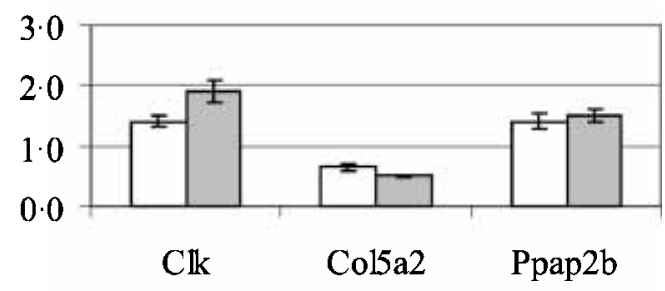

Lung

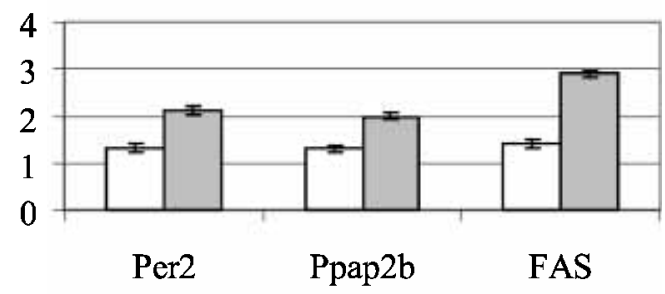

Heart

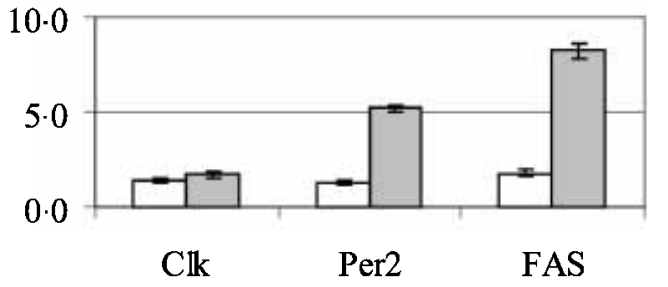

ment while expression of 7-dehydrocholesterol reductase and melanocortin 2 receptor (adrenocorticotropic hormone (ACTH) receptor) were induced (Fig. 5). The altered expression profiles of the majority of these genes were also verified by Q-PCR (Fig. 6).

It has been well documented that binding of ACTH to its receptor stimulates production of adrenal steroids (i.e. glucocorticoids and mineralocorticoids) from the adrenal cortex, rendering the ACTH receptor a key player in regulation of adrenal steroidogenesis (Beuschlein et al. 2001). We examined the expression of the ACTH receptor in the adrenal tissues by Q-PGR, and found that it was significantly induced $(P<0 \cdot 05$ by t test) upon LXR agonist treatment relative to the non-treated controls (Fig. 6). Measurements of corticosterone in the serum showed that treatment with the LXR agonist significantly increased corticosterone levels in serum $(P<0.05)$ in the wild-type mice but not in LXR $\alpha^{-/-} / \mathrm{LXR} \beta^{-/-}$mice (Fig. 7). However, serum levels of corticosteroids were five times higher in untreated $\mathrm{LXR} \alpha^{-/-} / \mathrm{LXR} \beta^{-/-}$mice compared with untreated wild-type mice $(P<0 \cdot 01)$, indicating that LXRs are likely to be involved both in maintaining basal levels of adrenal glucocorticoid production, and in controlling adaptive changes in glucocorticoid levels.

The surprising differences between serum levels of corticosteroids in wild-type mice versus $\mathrm{LXR} \alpha^{-/-} / \mathrm{LXR} \beta^{-/-}$mice prompted us to look at differences in expression profiles between these groups as well. A large number of genes showed altered expression levels in wild-type mice versus $\mathrm{LXR} \alpha^{-/-} / \mathrm{LXR} \beta^{-/-}$mice in both liver and adrenal (Fig. 8). More genes showed altered expression profiles in this comparison than observed upon treatment of an LXR agonist in

Figure 4 Verification of microarray data by Q-PCR. Q-PCR on selected genes from liver, kidney, adrenal, lung and heart from T1317-treated versus control wild-type mice was performed on the same RNA as used in the microarray experiments. RNA from four mice in each group was used (except liver, three mice) and average fold change between T1317-treated versus control wild-type mice compared with the results from the microarray experiment. Fold change observed in microarrays (white bars) and Q-PCR (grey bars) for each gene tested are arranged adjacently ( \pm S.E.M.). Fold changes of all Q-PCR-verified genes in the Figure were statistically significant $(P<0.05$ using $t$ test). 


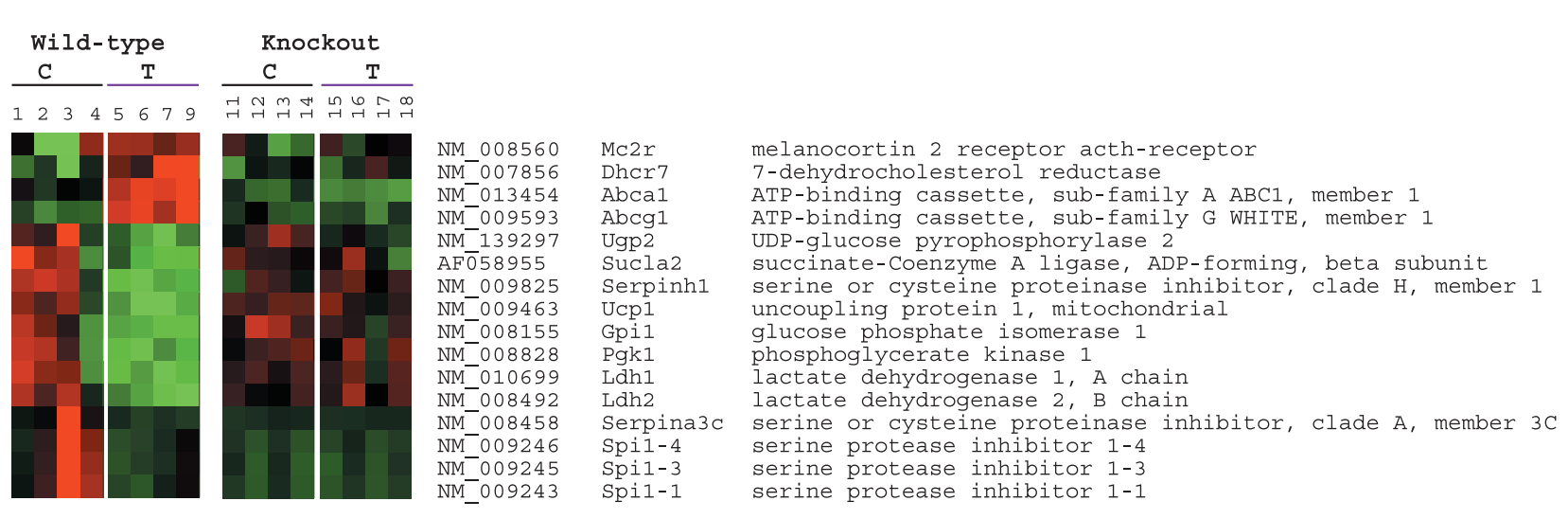

adrenals. The minority of the genes showed altered expression profiles in both comparisons. Hierarchical clustering analyses based on all genes with altered expression profiles $(P<0 \cdot 01)$ in wild-type mice versus $\mathrm{LXR} \alpha^{-/-} / \mathrm{LXR} \beta^{-/-}$mice in liver and adrenal are shown as supplementary Fig. 2A and B (see http://jme.endocrinology-journals.org/ content/vol33/issue3/), respectively.

The microarray data indicated a significant suppression of uncoupling protein (UCP)-1 in the adrenal tissues upon LXR agonist treatment. The UCPs are transporters that mediate proton leakage in mitochondria and are believed to be involved in thermogenesis and energy expenditure (Lowell and Spiegelman 2000). We therefore examined by Q-PCR the expression of several members of the UCP family (UCP-1-UCP-5) in the adrenals of mice with or without LXR agonist treatment. Fig. 9A shows that the expression of both UCP-1 and UCP-3 were significantly suppressed, by $22-$ and 2.5 -fold $(P<0.001$ and $P<0.05$ by t test), respectively, in adrenals from LXR-agonist-treated mice relative to their non-treated counterparts. Expression of UCP-2, -4 and -5 was induced slightly by the LXR agonist, but the inductions were not statistically significant. Thus, LXR appears to preferentially suppress UCP-1 and UCP-3 in adrenals.

Next we wanted to verify the observations from adrenals using the murine Y1 adrenocortical tumour cell line. Expression of both ABCAl (Fig. 9B) and ABCG1 (results not shown) was highly induced by treatment with LXR agonist $(P<0 \cdot 001)$. The induction was not inhibited by the presence of the protein-synthesis inhibitor cycloheximide, indicating a direct effect of LXR on ABCAl/G1 expression which is in accordance with previous reports. Both LXR $\alpha$ and LXR $\beta$ were expressed in this cell line (results not shown), further suggesting that the LXRs have a direct effect in adrenal. The Y1 cell line did not express UCP-1 so in order to investigate the effect of LXR on UCP-1 expression we used the murine HIB-1B preadipocyte cell line which was differentiated and treated with norepinephrine to express UCP-1 (Rabelo et al. 1997). Treatment with LXR agonist significantly reduced UCP-1 expression by $60 \%(P<0 \cdot 005$; Fig. $9 \mathrm{C})$. No inhibition of UCP-1 expression was observed by the treatment in the presence of cycloheximide. Hence, the effect of LXR needs protein synthesis for suppression, indicating an indirect effect of LXR on UCP-1 expression. 


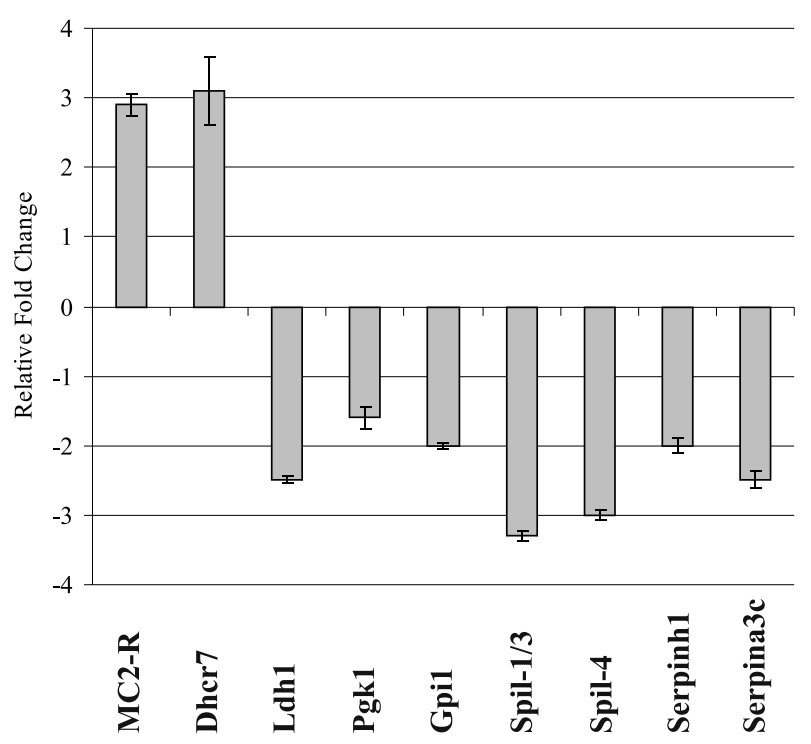

Figure 6 Verification of microarray data from adrenals by Q-PCR. Q-PCR was performed on selected genes from adrenal from T1317-treated versus control wild-type mice. RNA from four mice in each group was used and average relative fold changes from T1317-treated wild-type mice were compared with vehicle-treated wild-type mice whose average expression was set to 1. Error bars represent S.E.M.

\section{Discussion}

The LXRs are key players in various aspects of cholesterol, lipid and carbohydrate metabolism and, recently, the receptor isoforms have been suggested to mediate both anti-inflammatory and anti-atherosclerotic effects (Steffensen and Gustafsson 2004). Their biological functions have been elucidated mainly in liver, adipose tissue and macrophages, where most of their cognate target genes have been identified. In this study we show that the LXRs also play a role in several other tissues and identify putative LXR target genes indicating a role of the LXRs in various biochemical pathways like uncoupling, conjugation/dehydrogenation, phosphorylation/ dephosphorylation, transcription and protein turnover. These results suggest that the LXRs have a broader regulatory role than hitherto recognized, including control of energy metabolism, detoxification, signal transduction and protease activity.

The UCP family is located in mitochondria where they uncouple respiration from ATP synthesis, dissipating the proton gradient as heat. We observed that expression of both UCP-1 and
UCP-3 is highly suppressed in adrenals following treatment of wild-type mice with an LXR agonist. UCP-1 is considered to be expressed exclusively in brown adipose tissue while UCP-3 is expressed mostly in skeletal muscles (Del Mar GonzalezBarroso et al. 2000). To our knowledge, expression of UCPs has not previously been analysed in adrenals. A very low expression level of adipose fatty acid-binding protein (aFABP), an adiposetissue-specific protein, was detected in our adrenal samples. Hence, we cannot completely rule out the possibility of small contamination of RNA from adipose tissue in the RNA from adrenal. However, perirenal fat deposits are of white adipose tissue origin and should not express UCP-1 (Del Mar Gonzalez-Barroso et al. 2000), indicating that the monitored UCP-1 expression is adrenal-specific, a notion further supported by the Q-PGR detection of UCP-1 expression in commercially available RNA from human adrenals (results not shown). Both UCP-1 and UCP-3 have been suggested to be involved in energy expenditure (Del Mar GonzalezBarroso et al. 2000, Schrauwen 2002). Additionally, UCP-3 increases glucose uptake and synthesis of GLUT4, the major insulin-sensitive glucose transporter in muscle (Huppertz et al. 2001), and is involved in the flux of lipid substrates across the mitochondrial membrane (Dulloo \& Samec 2001). UCP-1 has also been suggested to transport fatty acids across the mitochondrial membrane (Garlid et al. 2000). These data suggest a novel role in which the LXRs are involved in the control of transport of lipids and carbohydrates, not only as inducers or suppressors of target genes involved in lipid biosynthesis and glucose production (i.e. gluconeogenesis). Also, most significantly, these observations suggest that LXR might be involved in control of energy expenditure.

One of the most intriguing findings was the upregulation of the ACTH receptor by the LXR agonist in adrenals. The production of steroid hormones in the adrenal cortex is regulated predominantly by ACTH which acts via its transmembrane receptor (MC2-R/ACTHreceptor) to turn on intracellular signalling cascades (Beuschlein et al. 2001). ACTH is a product of the pro-opiomelanocortin (POMC) gene that is primarily expressed in the pituitary gland, but its expression has been detected in numerous other tissues (Smith \& Funder 1988), whereas the ACTH receptor is mainly expressed in the adrenal cortex 


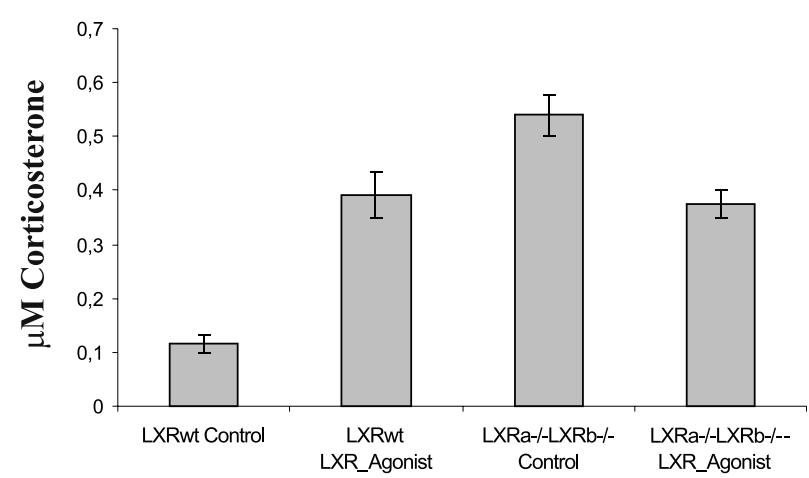

Figure 7 LXR induces corticosterone levels in serum.

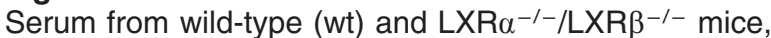
T1317-treated and controls, used in this study were collected before the mice were killed. Corticosterone levels were measured in serum from each individual mouse. The mean concentrations of the mice within each group are presented \pm S.E.M. The levels of significance of differences between the means were determined between wild-type control mice and wild-type LXR-agonist-treated mice $(P<0.04)$ and between non-treated wild-type mice and

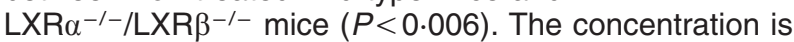
given as $\mu \mathrm{M}$ corticosterone in serum.

(Beuschlein et al. 2001). ACTH receptor expression was induced 3-fold by LXR agonist treatment in wild-type mice. In parallel, there was a 4-fold higher serum concentration of corticosterone in LXR-agonist-treated wild-type animals. There was a direct effect of LXR in Y1 cells as demonstrated by induced ABCA1 expression upon treatment with an LXR agonist. Y1 cells, which originate from adrenal cortex, expresses both the LXRs, further indicating a direct effect of the LXR in adrenal cortex. However, expression of the ACTH receptor was not altered upon treatment of an LXR agonist in Y1 cells. This observation also speaks in favour of indirect mechanisms of LXR effects.

A 5-fold-higher serum concentration of corticosterone in untreated $\mathrm{LXR} \alpha^{-/-} / \mathrm{LXR} \beta^{-/-}$mice compared with untreated wild-type mice provides further evidence for the involvement of the LXRs in adrenal steroidogenesis. These results suggest a tight regulation of glucocorticoid production by the LXRs. Higher levels of corticosterone in the absence of the LXRs indicate that, when unactivated by exogenous ligand, these receptors suppress basal glucocorticoid production, but, in contrast, induce glucocorticoid production upon activation by an LXR agonist. Such a dual function of unactivated versus activated nuclear receptor is also seen in case of the thyroid hormone receptor (Koenig 1998), for example. Surprisingly, no difference was seen in expression levels of the ACTH receptor in wild-type versus $\operatorname{LXR} \alpha^{-/-}$/ $\mathrm{LXR} \beta^{-/-}$mice. The physiological mechanism behind the strongly upregulated serum corticosterone levels in the double-knockout animals is therefore unclear. Both 20(S)- and 22(R)hydroxycholesterol are early intermediates in adrenal steroid hormone production and also are among the most potent endogenous agonists of the LXRs (Janowski et al. 1996, Forman et al. 1997,
Liver

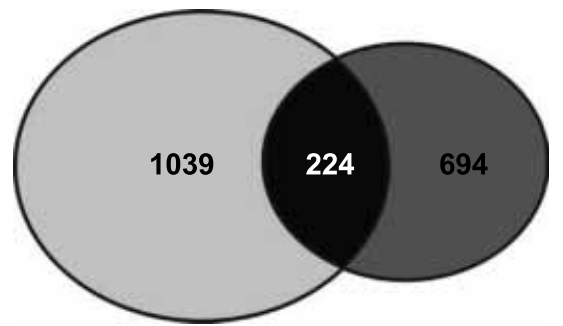

Adrenal

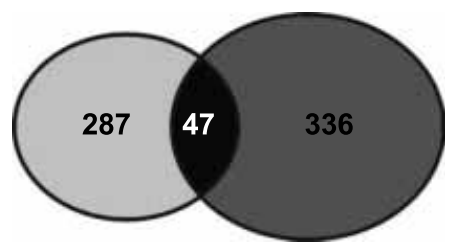

Figure 8 Venn diagram showing changes in expression pattern in wild-type versus $L X R \alpha^{-/-} / \mathrm{LXR} \beta^{-/-}$mice compared with LXR-responsive genes in wild-type mice. Genes were selected as described in the Materials and methods section under Data analysis $(P<0.01)$ in liver and adrenals. The number of genes responsive to treatment of an LXR agonist (light-grey shading) was compared with the number of genes with changed expression pattern in wild-type mice versus $L X R \alpha^{-/-} / \mathrm{LXR} \beta^{-/-}$mice (dark-grey shading). Genes with changed expression patterns in both groups were counted (black). 

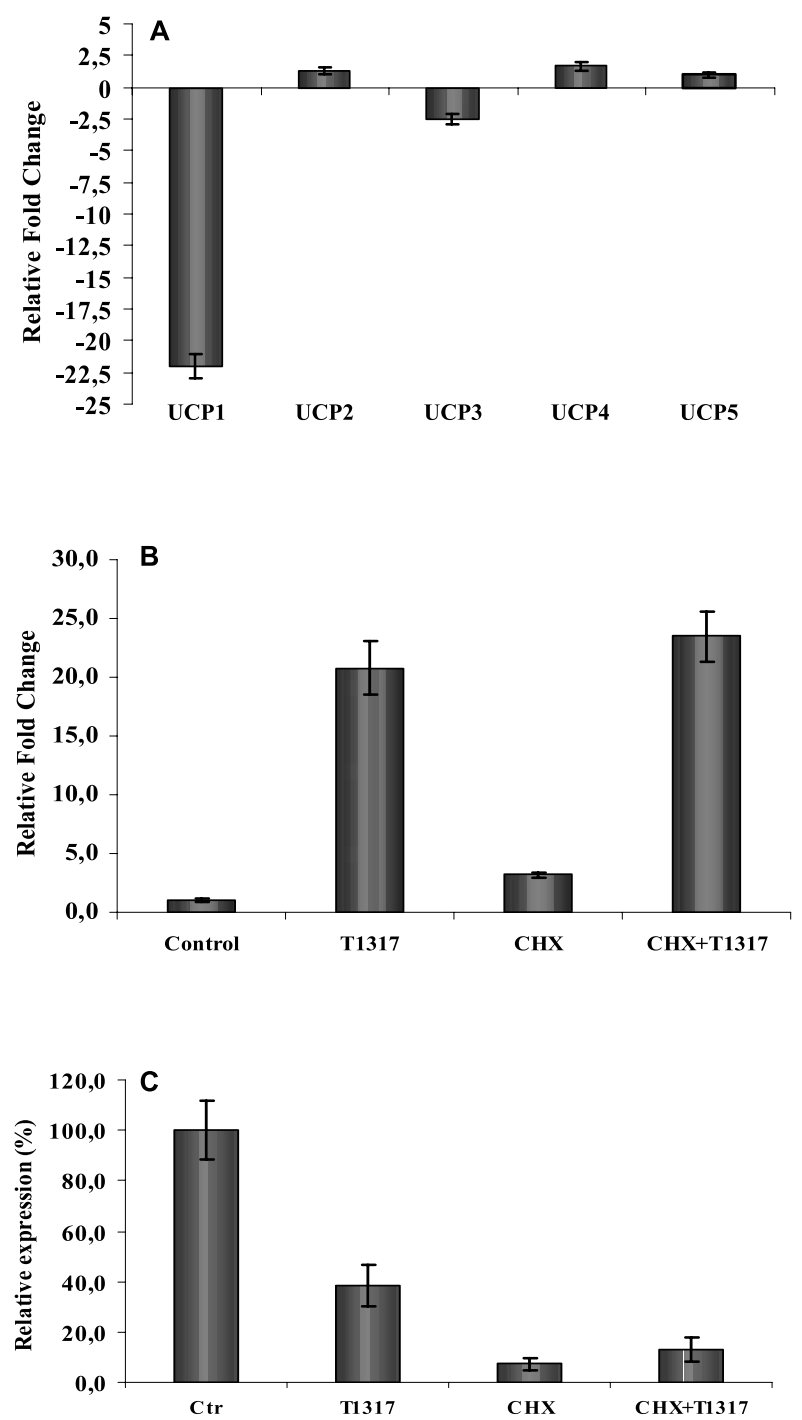

Figure 9 LXR suppresses expression of uncoupling proteins (UCPs). (A) Expression levels of the uncoupling family in adrenals were analysed by Q-PCR. RNA from four mice in each group was used and average relative fold changes from T1317-treated wild-type mice were compared with vehicle-treated wild-type mice whose average expression was set to 1. (B) Expression levels of $A B C A 1$ was analysed in the T1317-treated murine $Y 1$ adrenocortical tumour cell line in the presence or absence of the protein-synthesis inhibitor cycloheximide $(\mathrm{CHX})$. (C) Expression levels of UCP1 was analysed in the T1317-treated murine HIB-1B preadipocyte cell line after differentiation to brown adipocytes in the presence or absence of $\mathrm{CHX}$. Y1 and HIB-1B cells were treated for $24 \mathrm{~h}$ with $\mathrm{T} 1317$ or vehicle (DMSO) or pretreated with $1.25 \mu \mathrm{M} \mathrm{CHX}$ for 30 min before addition of $\mathrm{T} 1317$. Differentiated HIB-1B cells were given $1 \mu \mathrm{M}$ norepinephrine for $24 \mathrm{~h}$ before addition of $\mathrm{CHX}$ and T1317. Error bars represent S.E.M.
Lehmann et al. 1997). Hence, the adrenals contain endogenous LXR agonists, which, according to our observations, induce adrenal steroid hormone production (positive feed-forward control). We are currently investigating whether the stimulation of glucocorticoid production by the LXRs involves direct or indirect regulation of steroidogenic enzymes.

There is a growing body of evidence that serine proteases play an essential role in hormone biosynthesis in endocrine organs (Hill et al. 2002). Our data show that expression of several serine protease inhibitors in adrenals is suppressed by LXR agonist treatment including Spil-1, -3 and -4 (Figs 5 and 6). The functions of Spil protease inhibitors are poorly elucidated. They are suggested to consist of a five-member multigene family of anti-proteases with homologous functions to the human $\alpha 1$-anti-trypsin (Tardiff \& Krauter 1998). Spil-1 has moderate affinity to thrombin, and thrombin has been shown to increase aldosterone secretion from the adrenals (Raven et al. 2001). A family of G-protein-coupled receptors termed protease-activated receptors (PARs) has been identified that is activated by thrombin and trypsin (reviewed in Kawabata \& Kuroda 2000) and activation of PAR-1 stimulates aldosterone secretion from adrenals (Raven et al. 2001). The LXRs might suppress expression of protease inhibitors to prevent them from interfering with cleavage of, for example, thrombin and trypsin, thereby maintaining increased adrenal steroid synthesis. Expression of Spil-1, -3 and -4 was also reduced in $\mathrm{LXR} \alpha^{-/-} / \mathrm{LXR} \beta^{-/-}$mice compared with wild-type mice in adrenals (supplementary Fig. 2A), which could be an explanation to the increased serum corticosterone levels observed in $\mathrm{LXR} \alpha^{-/-} / \mathrm{LXR} \beta^{-/-}$mice, if our speculation above holds true. Also, the expression of serum/glucocorticoid-regulated kinase is induced in $\operatorname{LXR} \alpha^{-/-} / \mathrm{LXR} \beta^{-/-}$mice compared with wild-type mice (supplementary Fig. 2B), suggesting more than one plausible explanation to the altered corticosterone levels between these groups.

Furthermore, 7-dehydrocholesterol reductase, whose expression is induced by the LXR agonist in adrenals, is involved in cholesterol biosynthesis where it catalyses the reduction of 7dehydrocholesterol to cholesterol. Defects in this gene lead to impaired cholesterol biosynthesis 
and the gene has been linked to exaggerated $17 \alpha$-hydroxyprogesterone response to ACTH as well as adrenal hyperplasia, adrenal insufficiency, disorders of fetal adrenals, multiple congenital anomalies and mental retardation syndrome (see Porter 2000 and references therein). These results provide another line of evidence for a role for the LXRs in adrenal steroid production. Interestingly, a previous study in our laboratory showed that the LXRs suppress expression of $11 \beta$-hydroxysteroid dehydrogenase-1 (11 $\beta$-HSD-1) in vitro and in vivo (Stulnig et al. 2002a). This enzyme converts biologically inactive glucocorticoids to active hormones and is expressed abundantly in liver and adipose tissue (Bujalska et al. 1997). Thus LXRs appear to regulate not only glucocorticoid production but also local action of glucocorticoids in target tissues.

The difference in both corticosterone levels and expression profiles in wild-type mice versus $\mathrm{LXR} \alpha^{-/-} / \mathrm{LXR} \beta^{-/-}$mice indicates that the LXRs also serve a regulatory function of target genes when not activated. A study by Lala and colleagues (Hu et al. 2003) showed interaction between the LXRs and the corepressors SMRT (silent mediator of retinoic acid receptor and thyroid receptor) and $\mathrm{N}-\mathrm{CoR}$ (nuclear receptor corepressor), which was interrupted upon ligand binding. Both SMRT and N-CoR are known to inhibit gene expression when bound to nuclear receptors. A recent study by Wagner et al. (2003) showed that the LXRs interact with N-GoR and SMRT when not bound to ligand, repressing expression of ABCAl in macrophages. Dissociation or loss of the LXRs leads to derepression of ABCAl expression, suggesting that the LXRs work as suppressors of ABCAl expression when unliganded in agreement with our observation of significantly increased ABCAl expression in lung, heart and testis of $\mathrm{LXR} \alpha^{-/-} / \mathrm{LXR} \beta^{-/-}$mice (results not shown).

Discrepancies between the regulatory potential of the LXR isoforms have been reported that might be due to different affinity of the isoforms to an LXRE (LXR response element) (Feltkamp et al. 1999, Zhang et al. 2001, Li et al. 2002). Furthermore, PPAR $\alpha$ and PPAR $\gamma$ have been shown to compete with $\operatorname{LXR} \alpha$ for its heterodimer partner, RXR, abolishing binding of LXR $\alpha$ to the LXRE (Yoshikawa et al. 2003). Our observations further strengthen the notion that the regulatory mechanisms of the LXRs might be more complex than anticipated previously.

Only a few putative LXR target genes were found in brain and testis. Both tissues have a barrier against the circulating blood system providing protection from passive diffusion of compounds into these tissues, suggesting that this barrier might have been an obstacle for the entry of the T1317 compounds in these tissues. Expression of numerous genes was affected by T1317 treatment in brain and testis from $\mathrm{LXR}^{-/-}$/ $\mathrm{LXR} \beta^{-/-}$mice, contradicting the theory that the barrier does not allow entry of the T1317 compound in these tissues. Although speculative, it might be that the barrier in $\operatorname{LXR} \alpha^{-/-}$/ $\mathrm{LXR} \beta^{-/-}$mice is somehow disrupted, facilitating the entry of the T1317 compound which then exerts its non-LXR mediated effects.

Finally, our study indicates that treatment with an LXR agonist leads to suppressed expression of several glycolytic genes in adrenal. We have previously observed similar results in white and brown adipose tissue (Stulnig et al. 2002b) where we postulated that the LXRs mediate reduced glycolysis in extrahepatic tissues which is in agreement with this study. The many reports of the roles of LXRs in lipid and carbohydrate metabolism have led to expectations that LXRs could serve as drug targets for treatment of metabolic disorders (Steffensen \& Gustafsson 2004). The suppression of PEPCK expression (Stulnig et al. 2002a), leading to inhibition of gluconeogenesis, and reduced plasma glucose levels (Cao et al. 2003) mediated by an LXR agonist could mediate beneficial effects in diabetes mellitus type 2 . On the other hand, induction of lipogenesis leading to hypertriglyceridaemia as well as the observations made in this study where increased production of glucocorticoids was seen, indicate that the LXRs interfere with metabolic regulation in a much more complex manner than anticipated previously.

\section{Acknowledgements}

This research was supported by the Swedish Society for Medical Research (no. A200300834; to K R S), a Marie Curie Fellowship of the European Community programme Human Potential under contract number HPMF-CT-2000-00898 (to T M S), the Swedish Science Council, KaroBio 
$\mathrm{AB}$, and the Agency for Science, Technology and Research of Singapore. The European Commission is not responsible for any views or results expressed.

\section{References}

Alberti S, Schuster G, Parini P, Feltkamp D, Diczfalusy U, Rudling M, Angelin B, Bjorkhem I, Pettersson S \& Gustafsson J-Å 2001 Hepatic cholesterol metabolism and resistance to dietary cholesterol in LXRbeta-deficient mice. Fournal of Clinical Investigation $107565-573$.

Auboeuf D, Rieusset J, Fajas L, Vallier P, Frering V, Riou JP, Staels B, Auwerx J, Laville M \& Vidal H 1997 Tissue distribution and quantification of the expression of mRNAs of peroxisome proliferator-activated receptors and liver $\mathrm{X}$ receptor-alpha in humans no alteration in adipose tissue of obese and NIDDM patients. Diabetes 46 1319-1327.

Beuschlein F, Fassnacht M, Klink A, Allolio B \& Reincke M 2001 ACTH-receptor expression, regulation and role in adrenocortial tumor formation. European Fournal of Endocrinology 144 199-206.

Bujalska IJ, Kumar S \& Stewart PM 1997 Does central obesity reflect 'Cushing's disease of the omentum'? Lancet 349 1210-1213.

Cao G, Liang Y, Broderick CL, Oldham BA, Beyer TP, Schmidt RJ, Zhang Y, Stayrook KR, Suen C, Otto KA et al. 2003 Antidiabetic action of a liver $\mathrm{x}$ receptor agonist mediated by inhibition of hepatic gluconeogenesis. Fournal of Biological Chemistry 278 1131-1136.

Costet P, Luo Y, Wang N \& Tall AR 2000 Sterol-dependent transactivation of the $\mathrm{ABCl}$ promoter by the liver $\mathrm{X}$ receptor/retinoid X receptor. Fournal of Biological Chemistry $\mathbf{2 7 5}$ 28240-28245.

Dalen KT, Ulven SM, Bamberg K, Gustafsson J-Å \& Nebb HI 2003 Expression of the insulin responsive glucose transporter GLUT4 in adipocytes is dependent on liver X receptor. Fournal of Biological Chemistry 278 48283-48291.

Del Mar Gonzalez-Barroso M, Ricquier D \& Cassard-Doulcier AM 2000 The human uncoupling protein-1 gene (UCPl) present status and perspectives in obesity research. Obesity Reviews 1 61-72.

Dulloo AG \& Samec S 2001 Uncoupling proteins their roles in adaptive thermogenesis and substrate metabolism reconsidered. British Fournal of Nutrition 86 123-139.

Eberwine J, Spencer C, Miyashiro K, Mackler S \& Finnell R 1992 Complementary DNA synthesis in situ methods and applications. Methods in Enzymology 216 80-100.

Eisen MB, Spellman PT, Brown PO \& Botstein D 1998 Cluster analysis and display of genome-wide expression patterns. PNAS 95 14863-14868.

Feltkamp D, Wiebel FF, Alberti S \& Gustafsson J-Å 1999 Identification of a novel DNA binding site for nuclear orphan receptor OR1. Fournal of Biological Chemistry 274 10421-10429.

Forman BM, Ruan B, Chen J, Schroepfer GJ Jr \& Evans RM 1997 The orphan nuclear receptor LXRalpha is positively and negatively regulated by distinct products of mevalonate metabolism. PNAS 94 10588-10593.

Garlid KD, Jaburek M, Jezek P \& Varecha M 2000 How do uncoupling proteins uncouple? Biochimica Biophysica Acta 1459 383-389.

Hill RM, Coates LC, Parmar PK, Mezey E, Pearson JF \& Birch NP 2002 Expression and functional characterization of the serine protease inhibitor neuroserpin in endocrine cells. Annals of the New York Academy of Sciences 971 406-415.

Hu X, Li S, Wu J, Xia C \& Lala DS 2003 Liver x receptors interact with corepressors to regulate gene expression. Molecular

Endocrinology 17 1019-1026.
Huppertz C, Fischer BM, Kim YB, Kotani K, Vidal-Puig A, Slieker LJ, Sloop KW, Lowell BB \& Kahn BB 2001 Uncoupling protein 3 (UCP3) stimulates glucose uptake in muscle cells through a phosphoinositide 3-kinase-dependent mechanism. Fournal of Biological Chemistry 276 12520-12529.

Janowski BA, Willy PJ, Devi TR, Falck JR \& Mangelsdorf DJ 1996 An oxysterol signalling pathway mediated by the nuclear receptor LXR alpha. Nature 383 728-731.

Juvet LK, Andresen SM, Schuster GU, Dalen KT, Tobin KA, Hollung K, Haugen F, Jacinto S, Ulven SM, Bamberg K et al. 2003 On the role of liver $\mathrm{x}$ receptors in lipid accumulation in adipocytes. Molecular Endocrinology 17 172-182.

Kawabata A \& Kuroda R 2000 Protease-activated receptor (PAR), a novel family of $G$ protein-coupled seven trans-membrane domain receptors activation mechanisms and physiological roles. Fapanese Fournal of Pharmacology 82 171-174.

Kennedy MA, Venkateswaran A, Tarr PT, Xenarios I, Kudoh J, Shimizu N \& Edwards PA 2001 Characterization of the human ABGGl gene liver $\mathrm{X}$ receptor activates an internal promoter that produces a novel transcript encoding an alternative form of the protein. Fournal of Biological Chemistry $\mathbf{2 7 6}$ 39438-39447.

Koenig RJ 1998 Thyroid hormone receptor coactivators and corepressors. Thyroid 8 703-713.

Laffitte BA, Chao LC, Li J, Walczak R, Hummasti S, Joseph SB, Castrillo A, Wilpitz DC, Mangelsdorf DJ, Collins JL et al. 2003 Activation of liver $\mathrm{X}$ receptor improves glucose tolerance through coordinate regulation of glucose metabolism in liver and adipose tissue. PNAS 100 5419-5424.

Lehmann JM, Kliewer SA, Moore LB, Smith-Oliver TA, Oliver BB, Su JL, Sundseth SS, Winegar DA, Blanchard DE, Spencer TA \& Willson TM 1997 Activation of the nuclear receptor LXR by oxysterols defines a new hormone response pathway. Fournal of Biological Chemistry 272 3137-3140.

Li Y, Bolten C, Bhat BG, Woodring-Dietz J, Li S, Prayaga SK, Xia C \& Lala DS 2002 Induction of human liver X receptor alpha gene expression via an autoregulatory loop mechanism. Molecular Endocrinology 16 506-514.

Lowell BB \& Spiegelman BM 2000 Towards a molecular understanding of adaptive thermogenesis. Nature 404 652-660.

Mak PA, Laffitte BA, Desrumaux C, Joseph SB, Curtiss LK, Mangelsdorf DJ, Tontonoz P \& Edwards PA 2002 Regulated expression of the apolipoprotein E/C-I/C-IV/C-II gene cluster in murine and human macrophages. A critical role for nuclear liver $\mathrm{X}$ receptors alpha and beta. Fournal of Biological Chemistry 277 31900-31908.

Porter FD 2000 RSH/Smith-Lemli-Opitz syndrome a multiple congenital anomaly/mental retardation syndrome due to an inborn error of cholesterol biosynthesis. Molecular Genetics and Metabolism 71 163-174.

Rabelo R, Camirand A \& Silva JE 1997 3',5'-cyclic adenosine monophosphate-response sequences of the uncoupling protein gene are sequentially recruited during darglitazone-induced brown adipocyte differentiation. Endocrinology 138 5325-5332.

Raven PW, Kapas S, Carroll M \& Hinson JP 2001 Aldosterone secretion by the rat adrenal cortex is stimulated by the activation of protease-activated receptor 1. Fournal of Endocrinology 169 581-585.

Repa JJ, Turley SD, Lobaccaro JA, Medina J, Li L, Lustig K, Shan B, Heyman RA, Dietschy JM \& Mangelsdorf DJ 2000 Regulation of absorption and ABC1-mediated efflux of cholesterol by RXR heterodimers. Science 289 1524-1529.

Repa JJ, Berge KE, Pomajzl C, Richardson JA, Hobbs H \& Mangelsdorf DJ 2002 Regulation of ATP-binding cassette sterol transporters ABCG5 and ABCG8 by the liver X receptors alpha and beta. Fournal of Biological Chemistry 277 18793-18800. 
Schrauwen P 2002 Skeletal muscle uncoupling protein 3 (UCP3) mitochondrial uncoupling protein in search of a function. Current Opinion in Clinical Nutrition and Metabolic Care 5 265-270.

Schuster GU, Parini P, Wang L, Alberti S, Steffensen KR, Hansson GK, Angelin B \& Gustafsson J-Å 2002 Accumulation of foam cells in liver X receptor-deficient mice. Circulation 106 1147-1153.

Smith AI \& Funder JW 1988 Proopiomelanocortin processing in the pituitary, central nervous system, and peripheral tissues. Endocrine Reviews 9 159-179.

Sotiriou C, Powles TJ, Dowsett M, Jazaeri AA, Feldman AL, Assersohn L, Gadisetti C, Libutti SK \& Liu ET 2002 Gene expression profiles derived from fine needle aspiration correlate with response to systemic chemotherapy in breast cancer. Breast Cancer Research 4 R3.

Steffensen KR \& Gustafsson J-A 2004 Putative metabolic effects of the liver X receptor (LXR). Diabetes 53 Suppl 1 S36-S42.

Stulnig TM, Oppermann U, Steffensen KR, Schuster GU \& Gustafsson J-A 2002a Liver X receptors downregulate 11 beta-hydroxysteroid dehydrogenase type 1 expression and activity. Diabetes 51 2426-2433.

Stulnig TM, Steffensen KR, Gao H, Reimers M, Dahlman-Wright K, Schuster GU \& Gustafsson J-A 2002b Novel roles of liver X receptors exposed by gene expression profiling in liver and adipose tissue. Molecular Pharmacology 62 1299-1305.

Tardiff J \& Krauter KS 1998 Divergent expression of alphal-protease inhibitor genes in mouse and human. Nucleic Acids Research 26 3794-3799.

Wagner BL, Valledor AF, Shao G, Daige CL, Bischoff ED, Petrowski M, Jepsen K, Baek SH, Heyman RA, Rosenfeld MG et al. 2003 Promoter-specific roles for liver X receptor/corepressor complexes in the regulation of ABCAl and SREBPl gene expression. Molecular and Cellular Biology 23 5780-5789.

Willy PJ, Umesono K, Ong ES, Evans RM, Heyman RA \& Mangelsdorf DJ 1995 LXR, a nuclear receptor that defines a distinct retinoid response pathway. Genes and Development $\mathbf{9}$ 1033-1045.

Yoshikawa T, Ide Ti, Shimano H, Yahagi N, Amemiya-Kudo M, Matsuzaka T, Yatoh S, Kitamine T, Okazaki H, Tamura Y et al. 2003 Cross-talk between peroxisome proliferator-activated receptor (PPAR) and liver X receptor (LXR) in nutritional regulation of fatty acid metabolism. I. PPARs suppress sterol regulatory element binding protein-1c promoter through inhibition of LXR signaling. Molecular Endocrinology 17 1240-1254.

Zhang Y, Repa JJ, Gauthier K \& Mangelsdorf DJ 2001 Regulation of lipoprotein lipase by the oxysterol receptors, LXRalpha and LXRbeta. Fournal of Biological Chemistry 276 43018-43024.

Received 21 July 2004

Accepted 17 August 2004 